\title{
Effect of phenylephrine/ketorolac on iris fixation ring use and surgical times in patients at risk of intraoperative miosis
}

This article was published in the following Dove Press journal:

Clinical Ophthalmology

\section{Denise Visco}

Eyes of York Cataract \& Laser Center, York, PA, USA
Correspondence: Denise Visco

Eyes of York Cataract \& Laser Center,

I 800 Kenneth Road, Suite I, York,

PA 17408, USA

Tel +I 7177672000

Fax+I 7177672013

Email dvisco@eyesofyork.com
Purpose: To evaluate the effect of intracameral phenylephrine/ketorolac (1\%/0.3\%) during cataract surgery on the use of iris fixation ring and surgical time in patients with poor pupil dilation $(\leq 5.0 \mathrm{~mm}$ ) or intraoperative floppy iris syndrome (IFIS).

Setting: Private practice outpatient surgical center.

Design: This retrospective analysis was conducted from January 1, 2014 to October 7, 2015. Materials and methods: The use of iris fixation rings was evaluated in a retrospective analysis of 46 patients who underwent cataract surgery from January 1, 2014, to October 7, 2015, and who were identified before surgery to be at risk for intraoperative miosis. The qualifying factors were presurgical examination of pupil dilation $\leq 5.0 \mathrm{~mm}$ after being administered topical tropicamide $1 \%$ and phenylephrine $2.5 \%$ or history of IFIS during surgery in the fellow eye. All patients received a 2-day preoperative course of topical nonsteroidal anti-inflammatory drugs (NSAIDs) and day-of-surgery preoperative dilation using topical cyclopentolate $1 \%$, tropicamide $1 \%$, and phenylephrine 10\%. Phenylephrine/ketorolac 1\%/0.3\% $\left(\mathrm{Omidria}^{\circledR}\right)$ or epinephrine 1:1,000 with sulfites was added to the ophthalmic irrigation solution and delivered intracamerally at the start of the procedure and throughout surgery. The use of iris fixation rings and surgical time for each patient were captured for each group.

Results: Eighteen (50\%) of the patients in the epinephrine group and no patients in the phenylephrine/ketorolac group required iris fixation ring insertion to maintain pupil dilation or to control IFIS ( $p=0.0034$ ). Mean surgical time was significantly shorter in the group of patients who received phenylephrine/ketorolac $(p=0.0068)$.

Conclusion: In this retrospective cohort analysis of patients with poorly dilated pupils and/or IFIS, the use of intracameral phenylephrine/ketorolac in patients at risk for intraoperative miosis resulted in significantly less iris fixation ring use and significantly shorter surgical time when compared with intracameral epinephrine use.

Keywords: cataract extraction, miosis, phenylephrine, ketorolac, iris fixation ring, Malyugin ring, OMS302, Omidria ${ }^{\circledR}$

\section{Synopsis}

This retrospective analysis shows that, compared with intracameral epinephrine, intracameral phenylephrine/ketorolac $(1 \% / 0.3 \%)$ reduces the need for iris fixation rings and shortens the surgical time in cataract patients at risk for intraoperative miosis.

\section{Introduction}

In the United States, an estimated $50 \%$ of cases of low vision are attributable to agerelated cataract. ${ }^{1}$ In most patients, cataract surgery is successful in restoring vision 
and in significantly improving daily functioning and quality of life..$^{2-4}$ The overall risk of surgical complications is low, with any single complication estimated to affect fewer than $5 \%$ of cataract patients overall. However, when miosis occurs during surgery, the surgical field is restricted, and the risk of surgical complications such as tissue damage, retained lens material, and vitreous loss is increased. ${ }^{5-8}$ Patient-specific factors including diabetes mellitus, pseudoexfoliation, and intraoperative floppy iris syndrome (IFIS) are also known to increase the risk of intraoperative miosis or inadequate pupil dilation. ${ }^{6,-11}$

When intraoperative miosis occurs, the surgeon has a choice in pupil management. Typically, the first approach is intracameral injection of a pharmaceutical mydriatic agent such as epinephrine or phenylephrine or injection of ophthalmic viscoelastic devices to enhance mydriasis. ${ }^{12,13}$ If miosis persists, the surgeon may choose to insert a pupil expansion device to maintain adequate pupil size or manually stretch the pupil to manipulate the surgical field. ${ }^{12-14}$ The use of devices or surgical techniques to maintain mydriasis can lead to iris damage and other surgical complications.

The purpose of this study was to examine the effect of intracameral phenylephrine/ketorolac compared to intracameral epinephrine on the frequency of iris fixation ring use and on surgical time in patients identified to be at risk for intraoperative miosis before surgery.

\section{Materials and methods}

This retrospective analysis was conducted from January 1, 2014, to October 7, 2015, and included cataract surgery patients identified to be at risk for intraoperative miosis before surgery and, therefore, anticipated to require the usage of an iris fixation ring to maintain adequate surgical visualization and pupil management. The inclusion criteria were 1) patients who were required to have a pupil dilation of $\leq 5.0 \mathrm{~mm}$ with topical mydriatics at presurgical examination or 2) a history of IFIS during cataract surgery in the fellow eye. Patients undergoing routine cataract surgery consultation were dilated with tropicamide $1 \%$ and phenylephrine $2.5 \%$ and underwent fundus examination 20-30 minutes later. Patients with dilated pupils $\leq 5.0 \mathrm{~mm}$, determined using a 5 -mm light beam over the pupil diameter, were flagged as possible complex surgical cases. For patients in whom the operating surgeon had difficulty with IFIS in the first operative eye, the second eye was flagged as "possible complex" on the surgical schedule. IRB approval and patient written informed consent were not sought as our institution allows for the secondary use of patient data without patient identification and reporting of only composite data.
The following data were captured from the medical records of each patient: sex, age, axial length, date of surgical procedure, surgical time, use of an iris fixation ring, use of femtosecond laser, history of alpha-1 antagonist medication use, and agent added to the ophthalmic irrigation solution (ie, epinephrine or phenylephrine/ketorolac).

All patients received a 2-day preoperative course of topical nonsteroidal anti-inflammatory drugs (NSAIDs) and day-of-surgery preoperative dilation using topical cyclopentolate $1 \%$, tropicamide $1 \%$, and phenylephrine $10 \%$. Based on the insurance coverage, either phenylephrine/ketorolac (1\%/0.3\%, Omidria ${ }^{\circledR}$; Omeros Corporation, Seattle, WA, USA) or epinephrine 1:1,000 w/sulfites were added to the ophthalmic irrigation solution and delivered intracamerally at the start of the procedure and in the irrigating solution throughout the surgery. All procedures were performed by the same surgeon (DV).

\section{Statistical analysis}

Summary statistics were calculated to describe patient demographic information for each group, including mean and median patient age, percentage of male patients, mean axial length, and number of patients with a history of alpha-1 antagonist use. Due to small sample size, Wilcoxon rank-sum or Fisher's exact tests, as appropriate, were used to compare summary statistics between phenylephrine/ ketorolac and epinephrine groups. Mean surgical times were compared between the group receiving phenylephrine/ ketorolac, epinephrine with no ring use, and epinephrine with ring use, with a Wilcoxon rank-sum test. The Bonferroni method was used for multiple comparisons. Statistical significance was defined as a $p<0.05$. Statistical analyses were performed using Stata v. 11.0 (StataCorp, College Station, TX, USA).

\section{Results}

A total of 46 cataract surgery patients at risk for intraoperative miosis were included in this study. Patients received either phenylephrine/ketorolac $(n=10)$ or epinephrine 1:1,000 with sulfites $(n=36)$ in the ophthalmic irrigation solution administered intracamerally at the start of surgery and in the ophthalmic irrigation solution throughout the procedure. All patients underwent standard phacoemulsification except for two patients in the epinephrine group on whom femtosecond laser-assisted cataract surgery was performed. Demographic characteristics were similar between groups (Table 1). In the phenylephrine/ketorolac group, the mean age was 75.5 years and $50 \%$ of patients were male. In the epinephrine group, the mean age was 79.1 years and $47 \%$ of patients were male. 
Table I Patient demographic characteristics

\begin{tabular}{|c|c|c|c|}
\hline & $\begin{array}{l}\text { Phenylephrine/ } \\
\text { ketorolac } \\
(\mathrm{n}=10)\end{array}$ & $\begin{array}{l}\text { Epinephrine } \\
(n=36)\end{array}$ & $p$-value \\
\hline $\begin{array}{l}\text { Age (years), mean } \\
\text { (range), median }\end{array}$ & 75.7 (66-85), 77 & 79.1 (57-95), 8I.5 & $0.0848^{a}$ \\
\hline Male, n (\%) & $5(50)$ & $17(47)$ & $1.000^{\mathrm{b}}$ \\
\hline $\begin{array}{l}\text { Axial length }(\mathrm{mm}) \text {, } \\
\text { mean (SD) }\end{array}$ & $23.26(0.86)$ & $23.5 \mathrm{I}(0.98)$ & $0.497^{\mathrm{a}}$ \\
\hline $\begin{array}{l}\text { Alpha-I antagonist } \\
\text { use, } n(\%)\end{array}$ & 0 & $6(17)$ & $0.315^{b}$ \\
\hline
\end{tabular}

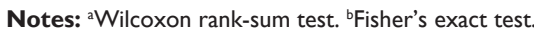

Iris fixation rings were used in 18 patients in the epinephrine group (50\%) compared to no rings used in the phenylephrine/ketorolac group (Table $2 ; p=0.0034$ ). Of the six patients in the epinephrine group who had a history of alpha-1 antagonist use, four (67\%) required an iris fixation ring during surgery, a rate slightly higher than the overall rate of iris fixation ring use in the epinephrine group. The mean surgical time was significantly shorter in patients receiving phenylephrine/ ketorolac compared to those receiving epinephrine (Table 2; 10.1 and 14.3 minutes, respectively; $p=0.0068$ ).

In the epinephrine group, among patients who did not require an iris fixation ring, the mean surgical time $( \pm \mathrm{SD})$ was $11.6( \pm 3.45)$ minutes, while, among epinephrine patients who required an iris fixation ring, the mean surgical time was 17.1 $( \pm 6.51)$ minutes (Figure 1). The mean surgical time for the phenylephrine/ketorolac group was numerically shorter than that for the group receiving epinephrine with no iris fixation ring, although the difference was not statistically significant (10.1 vs 11.6 minutes, respectively). The mean surgical time for the phenylephrine/ketorolac group was significantly shorter than that for the group receiving epinephrine who also required an iris fixation ring ( 10.1 vs 17.1 minutes; $p=0.0002$ ). Similarly, the mean surgical time for the group receiving epinephrine and no fixation ring was significantly shorter than that for the group requiring rings ( 11.6 vs 17.1 minutes; $p=0.0042$ ).

\section{Discussion}

This was a retrospective analysis of patients with poorly dilated pupils ( $\leq 5.0 \mathrm{~mm}$ with topical tropicamide $1 \%$ and

Table 2 Surgical characteristics by treatment group

\begin{tabular}{|c|c|c|c|}
\hline & $\begin{array}{l}\text { Phenylephrine/ } \\
\text { ketorolac } \\
(\mathrm{n}=10)\end{array}$ & $\begin{array}{l}\text { Epinephrine } \\
(\mathrm{n}=36)\end{array}$ & $p$-value \\
\hline Ring use, n (\%) & 0 & $18(50)$ & $0.0034^{a}$ \\
\hline $\begin{array}{l}\text { Surgical time (minutes), } \\
\text { mean (range), median }\end{array}$ & $10.1(8-13), 10$ & I 4.3 (7-39), 13 & $0.0068^{b}$ \\
\hline
\end{tabular}

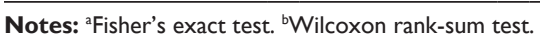

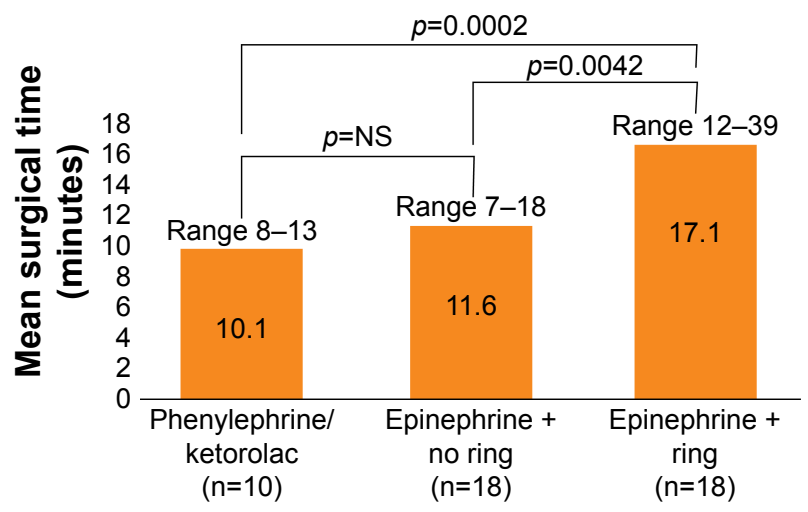

Figure I Mean surgical times for the group receiving phenylephrine/ketorolac, epinephrine without an iris fixation ring, and epinephrine with an iris fixation ring. Notes: No phenylephrine/ketorolac-treated patients required an iris fixation ring. Significance was tested with a Wilcoxon rank-sum test using the Bonferroni method to correct for multiple comparisons.

phenylephrine $2.5 \%$ ) or a known history of IFIS. Intracameral phenylephrine/ketorolac significantly reduced the need for iris fixation rings in patients at risk for intraoperative miosis. None of the patients receiving phenylephrine/ketorolac required iris fixation rings compared to $50 \%$ of patients receiving intracameral epinephrine.

The mean surgical time was significantly shorter for patients in the phenylephrine/ketorolac group than in the epinephrine group. An important factor in the difference in surgical times between these groups is the large percentage of epinephrine cases that required iris fixation rings. In comparing mean surgical times, an analysis was undertaken across three groups: patients receiving phenylephrine/ketorolac, patients receiving epinephrine who did not require an iris fixation ring, and patients receiving epinephrine who did require an iris fixation ring. There was a numerical but not statistically significant difference between the two groups in which rings were not used (ie, phenylephrine/ketorolac compared to epinephrine with no iris fixation ring use), but there was a pronounced difference in surgical times between the group in which iris fixation rings were used compared to each of the other two groups. There may be a variety of factors contributing to the shorter surgical times in the phenylephrine/ ketorolac group, and a larger sample size could help identify these factors.

A femtosecond laser was available for use according to the surgeon's routine clinical practice. In this study, all patients who underwent femtosecond laser-assisted surgery happened to be in the phenylephrine/ketorolac group. It has been shown that femtosecond laser treatment can cause miosis or result in a smaller pupil size at the time of phacoemulsification. ${ }^{15}$ Therefore, the use of femtosecond laser would likely increase the risk of requiring iris fixation 
rings in the phenylephrine/ketorolac group; however, in this study, none of the patients in that group required mechanical dilation.

The patients included in this study were those with known risk factors for intraoperative miosis, which may overestimate iris fixation ring use if attempting to generalize these results to a broad population of cataract surgery patients without these risk factors. It is notable that the results showing a lower rate of iris fixation rings and shorter surgical times in the phenylephrine/ketorolac group are consistent with other studies examining these factors. ${ }^{16,17}$ Other studies, conducted in the general cataract population, found that phenylephrine/ ketorolac is associated with lower rates of pupil expansion device use and shorter surgical times when surgical times are analyzed. All surgical procedures in this study were conducted by a single surgeon over a relatively short time frame, and all patients received the same preoperative medication regimen, so it is unlikely that there were treatment variations beyond the use of phenylephrine/ketorolac or epinephrine.

There were no patients with a history of alpha-1 antagonist use in the group receiving phenylephrine/ketorolac and six patients with a history of alpha-1 antagonist use in the epinephrine group (difference is not statistically significant). Of the six patients in the epinephrine group who did have a history of alpha-1 antagonist use, four (67\%) required an iris fixation ring during surgery. The rate of iris fixation ring use in these patients is only slightly higher than the rate in the epinephrine group overall. A larger sample size would be necessary to capture a more precise picture of patient risk characteristics and their effects on the use of pupil expansion devices.

\section{Conclusion}

In cataract surgery patients with known risk factors for intraoperative miosis, the use of intracameral phenylephrine/ ketorolac resulted in significantly reduced use of iris fixation rings compared to the use of intracameral epinephrine. This reduction in use of iris fixation rings would likely reduce the number of patients who experience complications due to mechanical pupil management. Furthermore, the surgeon's determination that iris fixation rings were not needed in these high-risk patients indicates that their pupils were manageable with phenylephrine/ketorolac alone, decreasing the risk of all complications associated with small pupils. Surgical times were also significantly shortened by an average of 2.9 minutes in patients who received phenylephrine/ ketorolac compared to those receiving epinephrine. Patients who required iris fixation rings also had significantly longer surgical times compared to those who did not require rings. Intracameral phenylephrine/ketorolac administered in the irrigation solution during cataract surgery may result in fewer complications due to pupil management and due to small pupils and may allow higher surgical throughput with overall shorter surgical procedure times.

\section{Acknowledgments}

Omeros Corporation provided medical writing support for this paper. This study was previously presented at the American Society of Cataract and Refractive Surgery 2016, New Orleans, LA, USA, Sunday, May 8, 2016, Paper Session 2-Q, \#22817.

\section{Disclosure}

Dr Visco is a consultant for Omeros Corporation. She has received honoraria or payments for consulting or speaking services for Bausch + Lomb, Inc., i-Optics, LENSAR, Inc., Omeros Corporation, RVO, Sun Ophthalmic, and Shire Plc. The author reports no other conflicts of interest in this work.

\section{References}

1. Congdon N, O'Colmain B, Klaver CCW, et al; Eye Diseases Prevalence Research Group. Causes and prevalence of visual impairment among adults in the United States. Arch Ophthalmol. 2004;122(4): 477-485.

2. Fraser ML, Meuleners LB, Lee AH, Ng JQ, Morlet N. Vision, quality of life and depressive symptoms after first eye cataract surgery. Psychogeriatrics. 2013;13(4):237-243.

3. Harrer A, Gerstmeyer K, Hirnschall N, et al. Impact of bilateral cataract surgery on vision-related activity limitations. $J$ Cataract Refract Surg. 2013;39:680-685.

4. Jaycock P, Johnston RL, Taylor H, et al. The Cataract National Dataset electronic multi-centre audit of 55567 operations: updating benchmark standards of care in the United Kingdom and internationally. Eye. 2007; 23:38-49.

5. Gonzalez-Gonzalez L, Grob S, Daly M. Management of mydriasis and pain in cataract and intraocular lens surgery: review of current medications and future directions. Clin Ophthalmol. 2014;8:1281-1289.

6. Greenberg PB, Tseng VL, Wu W-C, et al. Prevalence and predictors of ocular complications associated with cataract surgery in United States veterans. Ophthalmology. 2011;118(3):507-514.

7. Randleman JB, Wolfe JD, Woodward M, Lynn MJ, Cherwek DH, Srivastava SK. The resident surgeon phacoemulsification learning curve. Arch Ophthalmol. 2007;125(9):1215-1219.

8. Behndig A, Lundberg B, Bäckström G. Intracameral mydriatics in cataract surgery. In: Zaidi FH, editor. Cataract Surgery. InTech; 2013. Available from: https://www.intechopen.com/books/cataract-surgery/ intracameral-mydriatics-in-cataract-surgery. Accessed January 9, 2018.

9. Guzek JP, Holm M, Cotter JB, et al. Risk factors for intraoperative complications in 1000 extracapsular cataract cases. Ophthalmology. 1987; 94(5):461-466.

10. Vollman DE, Gonzalez-Gonzalez LA, Chomsky A, Daly MK, Baze E, Lawrence M. Intraoperative floppy iris and prevalence of intraoperative complications: results from ophthalmic surgery outcomes database. Am J Ophthalmol. 2014;157(6):1130-1135.e1. 
11. Zaczek A, Zetterström C. Cataract surgery and pupil size in patients with diabetes mellitus. Acta Ophthalmol Scand. 1997;75:429-432.

12. Hashemi H, Seyedian MA, Mohammadpour M. Small pupil and cataract surgery. Curr Opin Ophthalmol. 2015;26:3-9.

13. Assaf A. Cataract surgery in small pupils: choosing the appropriate tool. Cataract Refract Surg Today Eur. 2013:16-20. Available from: https://crstodayeurope.com/articles/2013-sep/cataract-surgery-insmall-pupils-online-survey-choosing-the-appropriate-tool/. Accessed January 9, 2018.

14. Akman A, Yilmaz G, Oto S, Akova YA. Comparison of various pupil dilatation methods for phacoemulsification in eyes with a small pupil secondary to pseudoexfoliation. Ophthalmology. 2004;111(9): 1693-1698.
15. Jun JH, Hwang KY, Chang SD, et al. Pupil-size alterations induced by photodisruption during femtosecond laser-assisted cataract surgery. $J$ Cataract Refract Surg. 2015;41:278-285.

16. Bucci FA, Fluet AT. A Comparison of the Frequency of the Use of the Malyugin(R) Ring with and without Intracameral Phenylephrine \& Ketorolac 1\%/0.3\% Injection at the Time of Routine Cataract Surgery. Seattle, WA: Association for Research in Vision and Ophthalmology; 2016.

17. Rosenberg E, Nattis A, Alevi D, et al. Initial experience, visual outcomes, and efficacy of intracameral phenylephrine and ketorolac $(1 \% / 0.3 \%)$ during cataract surgery. In: American Society of Cataract and Refractive Surgery Symposium \& Congress; New Orleans, LA; May 6-10, 2016.
Clinical Ophthalmology

\section{Publish your work in this journal}

Clinical Ophthalmology is an international, peer-reviewed journal covering all subspecialties within ophthalmology. Key topics include: Optometry; Visual science; Pharmacology and drug therapy in eye diseases; Basic Sciences; Primary and Secondary eye care; Patien Safety and Quality of Care Improvements. This journal is indexed on

Submit your manuscript here: http://www.dovepress.com/clinical-ophthalmology-journal

\section{Dovepress}

PubMed Central and CAS, and is the official journal of The Society of Clinical Ophthalmology (SCO). The manuscript management system is completely online and includes a very quick and fair peer-review system, which is all easy to use. Visit http://www.dovepress.com/ testimonials.php to read real quotes from published authors. 